\title{
Analysis on Occupational Well-being Status and Influencing Factors of Medical Staff in Tuberculosis Departments
}

\author{
Liyuan Wang, Xiuzheng Wang, Lingling Li, Shanshan Zhou, Yanling Li* \\ China Department of Tuberculosis, Affiliated Hospital of Hebei University, Baoding 071000, Hebei Province, China \\ *Corresponding author: Yanling Li, xsliyanling@163.com
}

\begin{abstract}
Objective: To investigate the status of occupational well-being of medical staff in tuberculosis department and analyze its influencing factors, so as to provide a basis for improving the occupational well-being of medical staff in tuberculosis department. Methods: In May 2020, we adopted the method of cluster sampling to select staff members from the tuberculosis departments of the Affiliated Hospital of Hebei University and infectious disease hospital. A total of 139 medical staff were recruited as the research subjects, and were investigated using medical staff occupational well-being scale. Results: The total score of occupational well-being was $76.46 \pm 8.97$ points, There were statistically significant differences $(P<0.05)$ in occupational well-being score among tuberculosis medical staff with different age, years of work, job title, night shift, marital status, and occupational type. The influencing factors of occupational well-being were the night shift, years of work, occupational type, and marital status $(P<0.05)$. Conclusion: The overall level of occupational well-being of tuberculosis medical staffs is moderate, and occupational well-being is affected by night shift, years of work, occupational type and marital status. It is recommended that managers take targeted measures to improve the occupational well-being of tuberculosis medical staff.
\end{abstract}

Keywords: Occupational well-being; Tuberculosis medical staff; Influencing factors

Publication date: July 2021; Online publication: July 30, 2021

\section{Introduction}

Occupational well-being is the continuously happy experience that the subject obtains based on the satisfaction of needs, the full play of potential, and the growth of strength when engaged in a certain profession. It is used to measure the positive emotion and cognitive evaluation of employees for work ${ }^{[1]}$. Studies have found that the occupational well-being of medical staff not only affects their physical and mental health, but also affects the quality of clinical care ${ }^{[2,3]}$. Due to the infectiousness of the tuberculosis ward, the working environment and the particularity of the work target, the tuberculosis medical staff are under tremendous pressure in the psychological, physiological, and social aspects ${ }^{[4]}$. However, literature on occupational well-being of tuberculosis medical staff remains scanty. This study aims to investigate the status quo of occupational well-being of tuberculosis medical staff and analyze its influencing factors. In order to take targeted intervention measures to improve the occupational well-being of tuberculosis medical staff, this could provide theoretical support and basis. 


\section{Subjects and methods}

\subsection{Research subjects}

Using the cluster sampling method, a total of 139 medical staff from 5 wards of the tuberculosis department of Baoding Affiliated Hospital of Hebei University and 3 wards of the Tuberculosis Department of Baoding Infectious Diseases Hospital were selected as the research subjects in May 2020. Inclusion criteria include: (i) medical staff working in the tuberculosis department during the investigation; (ii) clinical work in the tuberculosis department for $\geq 1$ year; (iii) informed consent. Exclusion criteria include: (i) individuals with mental illness or impaired consciousness; (ii) staff under advanced training or interns; (iii) medical personnel who cannot participate in the survey due to personal leave, sick leave, maternity leave, etc.

\subsection{Research tools}

\subsubsection{General information questionnaire}

General information questionnaire was used to collect data, the attributes of the hospital, gender, age, working years, education level, job title, night shift status, marital status, education level, occupation type, and whether or not the establishment of a career.

\subsubsection{Occupational well-being scale for medical workers}

The scale was designed and compiled by Hu Dongmei of Dalian Medical University in 2011 [5], and is mainly used to assess the level of occupational well-being of medical staff. The scale has 24 items and 5 dimensions, namely physical and mental health status (items 1-6), value/ability manifestation (items 7-12), social support (items 13-17), and economic income (items 18-20), and work environment (items 21-24). Each item adopts Likert 5-level scoring method with assigned score of 1-5 denoting the range from "completely non-conforming" to "completely conforming." Entries 1-6 are reverse entries and use reverse scoring. The total score is 24-120, of which 24-48 denotes low level, 48-96 denotes medium level, and 96120 denotes high level. The higher the score, the higher the level of occupational well-being of medical staff. After testing, the Cronbach's $\alpha$ coefficient of this scale is 0.904 .

\subsection{Investigation methods and quality control}

Using the questionnaire survey method, the surveyor explained the purpose of the survey and how to fill out the questionnaire form, and then sent the questionnaire to the medical staff to fill out by themselves, which was completed in about 5 minutes. In this survey, a total of 139 questionnaires were distributed and 139 were returned. The efficiency of the questionnaire was $100 \%$.

\subsection{Statistical processing}

SPSS statistical software version 22.0 was used for statistical analysis. General data of tuberculosis medical staff was expressed as mean, standard deviation, frequency and percentage. Multiple linear regression analysis was used to analyze the factors influencing the occupational well-being of tuberculosis medical staff in general data, and compare the scores of each dimension by $t$ test.

\section{Results}

\subsection{The status quo of medical personnel's occupational well-being}

The total average score of medical personnel's occupational well-being was $76.46 \pm 8.97$, and the total item average was 3.30, which was at a medium level. The overall feeling of current occupational well-being score is $66.43 \pm 5.04$ points. See Table 1 for details.

Among the 24 items, the 3 items with the highest scores are items 7, 11, and 8, with scores of $4.13 \pm 0.73$, $4.11 \pm 0.65$, and $4.08 \pm 0.71$, respectively. The three items with the lowest scores are items 3 , 1 , and 6 , with 
accompanying points of $2.19 \pm 0.82,2.41 \pm 1.25$, and $2.27 \pm 0.97$, respectively. See Table 2 and Table 3 for details.

Table 1. Scores of various dimensions of occupational well-being of medical staff $(n=139$, mean \pm SD)

\begin{tabular}{lcc}
\hline Dimension & Total score & The average score \\
\hline Value/ability manifestation & $19.03 \pm 4.11$ & $3.17 \pm 0.54$ \\
Social support & $18.83 \pm 4.23$ & $3.77 \pm 0.61$ \\
Work environment & $12.60 \pm 4.21$ & $3.15 \pm 0.58$ \\
Economic income & $8.42 \pm 4.13$ & $2.81 \pm 0.83$ \\
Physical and mental health status & $21.58 \pm 5.21$ & $3.60 \pm 0.64$ \\
\hline
\end{tabular}

Table 2. The 3 items with the highest scores for medical staff $(n=139)$

\begin{tabular}{llcc}
\hline Sort & \multicolumn{1}{c}{ Entry } & Dimension & Score \\
\hline 1 & 7. Do you think your profession is sacred & Value/ability manifestation & $4.13 \pm 0.73$ \\
2 & 11. Do you have a sense of accomplishment in your work & Value/ability manifestation & $4.11 \pm 0.65$ \\
3 & 8. Are you satisfied with your present working condition & Value/ability manifestation & $4.08 \pm 0.71$ \\
\hline
\end{tabular}

Table 3. The 3 items with the lowest scores for medical staff $(n=139)$

\begin{tabular}{lllc}
\hline Sort & \multicolumn{1}{c}{ Entry } & \multicolumn{1}{c}{ Dimension } & Score \\
\hline 1 & 3. At present, your work pressure is high & Physical and mental health status & $2.19 \pm 0.82$ \\
2 & 1. Your physical condition is often in sub-health state & Physical and mental health status & $2.27 \pm 0.97$ \\
3 & $\begin{array}{l}\text { 6. You feel very tired and have no time and energy to } \\
\text { do what you like }\end{array}$ & Physical and mental health status & $2.41 \pm 1.25$ \\
\hline
\end{tabular}

\subsection{Comparison of scores of medical staff's occupational well-being with different characteristics}

The results of this study showed that the difference in the total scores of medical staff's occupational wellbeing for different ages, working years, job titles, night shift status, marital status, and occupation type was statistically significant $(\mathrm{P}<0.05)$. See Table 4 for details.

\subsection{Multiple stepwise regression analysis of influencing factors of occupational well-being of medical staff}

The statistically significant items in the univariate analysis were used as independent variables, and the total score of occupational well-being was used as the dependent variable to conduct multiple stepwise regression analysis. Finally, the factors that enter the regression equation are as follows: night shift status, working years, occupation type, and marital status, which jointly explain $17.0 \%$ of the total variation of occupational happiness, indicating that the night shift status, working years, occupation type, and marital status of the medical staff are factors that affects their professional happiness (Table 5). 
Table 4. Comparison of occupational well-being scores of medical staff with different characteristics $(n=139)$

\begin{tabular}{|c|c|c|c|c|c|}
\hline Item & $\begin{array}{l}\text { Number of } \\
\text { cases }\end{array}$ & $\begin{array}{l}\text { Composition ratio } \\
(\%)\end{array}$ & $\begin{array}{l}\text { Occupational happiness } \\
\text { score (mean } \pm \text { SD) }\end{array}$ & $t / F$ value & $P$ value \\
\hline Hospital attributes & & & & -0.864 & 0.434 \\
\hline General Hospital & 91 & 65.5 & $77.11 \pm 8.17$ & & \\
\hline Specialist Hospital & 48 & 34.5 & $75.86 \pm 9.10$ & & \\
\hline Gender & & & & -0.527 & 0.636 \\
\hline Female & 92 & 66.2 & $75.41 \pm 9.17$ & & \\
\hline Male & 47 & 33.8 & $76.89 \pm 7.98$ & & \\
\hline Age & & & & 6.935 & $0.047 *$ \\
\hline$<$ or $=25$ years & 28 & 20.1 & $77.13 \pm 8.21$ & & \\
\hline $26-35$ & 69 & 49.7 & $74.22 \pm 9.11$ & & \\
\hline $36-45$ & 26 & 18.7 & $76.11 \pm 8.25$ & & \\
\hline$>46$ & 16 & 11.5 & $79.06 \pm 6.99$ & & \\
\hline Working years & & & & 17.273 & $0.000^{* *}$ \\
\hline 1 & 7 & 5.0 & $77.46 \pm 8.11$ & & \\
\hline $2-5$ & 37 & 26.6 & $74.29 \pm 8.91$ & & \\
\hline $6-10$ & 47 & 33.8 & $71.31 \pm 10.39$ & & \\
\hline $11-15$ & 25 & 18.0 & $72.16 \pm 9.99$ & & \\
\hline $16-20$ & 15 & 10.8 & $78.21 \pm 7.26$ & & \\
\hline$\geq 21$ & 8 & 5.8 & $80.03 \pm 7.09$ & & \\
\hline Education & & & & 1.913 & 0.615 \\
\hline Technical secondary school & 12 & 8.6 & $76.89 \pm 7.91$ & & \\
\hline Junior college & 20 & 14.4 & $75.02 \pm 9.93$ & & \\
\hline Undergraduate & 64 & 46.1 & $75.41 \pm 9.12$ & & \\
\hline Postgraduate and above & 43 & 30.9 & $77.89 \pm 7.28$ & & \\
\hline Job title & & & & 7.732 & $0.038 *$ \\
\hline Primary & 58 & 41.73 & $77.41 \pm 8.17$ & & \\
\hline Intermediate & 59 & 42.45 & $75.03 \pm 9.24$ & & \\
\hline Advanced & 22 & 15.83 & $78.89 \pm 7.18$ & & \\
\hline Night shift & & & & -4.160 & $0.000^{* *}$ \\
\hline Have & 112 & 80.58 & $74.63 \pm 9.29$ & & \\
\hline No & 27 & 19.42 & $78.83 \pm 7.71$ & & \\
\hline Marital status & & & & -3.935 & $0.000^{* *}$ \\
\hline Single & 23 & 16.55 & $74.63 \pm 9.32$ & & \\
\hline Married & 116 & 83.45 & $77.83 \pm 7.69$ & & \\
\hline Type of jobs & & & & -4.716 & $0.000^{* *}$ \\
\hline Doctors & 49 & 35.25 & $79.12 \pm 6.88$ & & \\
\hline Nurse & 90 & 64.75 & $74.63 \pm 10.01$ & & \\
\hline $\begin{array}{l}\text { Whether the business } \\
\text { establishment }\end{array}$ & & & & 2.036 & 0.563 \\
\hline Yes & 54 & 38.85 & $77.01 \pm 8.17$ & & \\
\hline No & 85 & 61.15 & $75.99 \pm 9.10$ & & \\
\hline
\end{tabular}


Table 5. Multiple linear regression analysis of occupational well-being of medical staff $(n=139)$

\begin{tabular}{lcccc}
\hline & Regression coefficient & Standardized regression coefficient & $\boldsymbol{t}$ & $\boldsymbol{P}$ \\
\hline Constant & 178.692 & - & 17.127 & 0.000 \\
Night shift & -9.645 & -0.142 & -5.741 & 0.000 \\
Working years & 7.568 & 0.141 & 3.889 & 0.000 \\
Type of jobs & -2.594 & -0.124 & -2.679 & 0.001 \\
Marital status & 2.733 & 0.055 & 2.323 & 0.002 \\
\hline
\end{tabular}

\section{Discussion}

\subsection{The status quo of occupational well-being of medical staff}

The total score of occupational well-being of medical staff in the tuberculosis department is $76.46 \pm 8.97$ points, which is lower than that in other related studies ${ }^{[6]}$, on the account of the following reasons: (i) Disease infectiousness: Doctors and nurses in the tuberculosis department often come into close contact with patients with infectious diseases and thus, face greater risks. If the immune resistance is weak or they catch colds, they are more likely to be infected with tuberculosis, so that medical staff are prone to tension, depression, pessimism and other emotions. (ii) High workload: At present, the incidence of tuberculosis is on the rise, and doctors and nurses engaged in tuberculosis care are severely lacking. In their work, tuberculosis nurses are not only responsible for implementing various treatments, but also paying attention to the mental health of patients. At the same time, they coordinate multiple relationships, such as the relationship with the patient, the relationship with the patient's family, and the relationship with the doctor. A series of problems have brought serious negative effects on the psychology and physiology of nurses in the tuberculosis department. (iii) Lack of spare time and difficulty in interpersonal communication: Due to its professional nature, the location of the tuberculosis department is relatively distant from others. The doctors and nurses do not interact much with the outside world, and they do not relax in their spare time. Depression will occur for a long time. (iv) Lack of mental health knowledge: Most doctors and nurses in the tuberculosis department have no or very little training in mental illness prevention knowledge, and usually cannot apply psychological expertise to deal with their own problems, leading to depression, low self-esteem, and mental fatigue.

The scores of each dimension of occupational well-being in this study are value/ability manifestation, social support, working environment, economic income, physical and mental health in order of higher professional orientation and better professional quality. In particular, it is necessary to realize that the realization of nurses' value orientation and goals determines occupational well-being at the same time. Therefore, the hospital's career planning should cover every nurse to maximize its value and make everyone feel valued [7]. The high score of social support indicates that the medical staff in the tuberculosis department in this study are working in a humanistic environment with high satisfaction. Tian Huishu et al. ${ }^{[8]}$ have proven that a well-cultured hospital environment promotes the team spirit and cohesion of medical staff, and at the same time improves the occupational well-being of medical staff. The working environment score is low, which may be related to current medical equipment. The relationship between doctors and patients is tense, and the hospital management and promotion system need to be improved ${ }^{[9]}$. The lowest score of physical and mental health may be directly related to the high infectiousness, high pressure, and high load of the tuberculosis department ${ }^{[10]}$. At the same time, they are faced with various disinfection methods and radiation damage, as well as various infections, such as hepatitis B, AIDS, and syphilis. It is reminded that managers should pay more attention to the physical and mental health of medical staff in the tuberculosis department and the working environment, improve the environment, and pay attention to physical and mental health to enhance professional well-being. 


\subsection{The impact of various factors on the occupational well-being of medical staff 4.2.1. Night shift situation}

This study shows that medical staff without night shifts have better occupational well-being than medical staff with night shifts, which is consistent with the results of Jin Xiaoyan ${ }^{[11]}$. The reasons are analyzed. On the one hand, nurses who often work night shifts have disordered work and rest schedules and irregular diets, leading to a series of sub-health problems, such as neurasthenia and stomach pain. On the other hand, compared with the day shift, the night shift has fewer people on duty, but the patients at night are usually in the emergent, critical, and severe states. Even in hospitalized patients, many emergencies such as cardiocerebrovascular emergencies usually occur at night, causing intense night work, increasing psychological pressure at work, and damaging the physical and mental health of nurses. Nurses who do not have night shifts have a relatively high quality of life and improved professional happiness.

\subsubsection{Working years}

The results of this study show that the occupational well-being scores of nurses with different working years are "low in the middle and high at both ends." Among them, medical staff who have worked for more than 16 years have accumulated certain work experience, social support and other aspects. They have demonstrated their ability at work, have considerable economic income, and stable work shifts, and can arrange their own lives according to their wishes after work, so their score of professional happiness is higher. For medical staff who have worked for 6-10 years and 11-15 years, their passion toward the work has long since faded, and they can't find an aspiration for the future. Work is more of a means to support their families; and in life, they are under a lot of pressure and have to deal with time conflict to take care of the family and educate children, and eventually, they may neglect to manage their own lifestyles. Therefore, their career happiness scores are low. Nursing managers should focus on the psychological needs of nurses at this stage ${ }^{[12]}$. Nurses who have worked for less than 5 years may enter the workplace because their work responsibilities are lighter than those of older nurses. They work with a learning mentality and have little psychological pressure. Compared with their student years, their financial income has improved. Entertainment and other aspects have been strengthened, and the professional happiness score is higher.

\subsubsection{Occupation type}

This study shows that doctors' occupational well-being is higher than that of nurses, which is consistent with the conclusions of Li Guihua's ${ }^{[13]}$ research. For a long time, the society's concept of focusing on medical care over nursing has caused many people to hold discrimination and prejudice against the profession of nurses. In the eyes of most patients and their families, nurses are just equivalent to caregivers or nanny, and doctors control their health or are the decision maker of survival. Because of this perspective, the nurses do not get the respect they deserve. In addition, the fear of infectious diseases from the outside makes the social status of nurses engaged in tuberculosis care lower ${ }^{[4]}$. Even among hospital staff, this concept of valuing medical care over nursing is still present. Doctors' sense of self-identity is significantly better than that of nurses. This is reflected in the fact that doctors score higher than nurses in the dimension of value or ability. Compared to doctors, nurses have fewer opportunities to continue their studies, and they cannot find the satisfaction and sense of accomplishment of self-worth at work. On the other hand, since doctors have a higher starting point and generally higher initial education qualifications than nurses; the higher their level of theoretical knowledge, operational skills, and scientific research capabilities, the more they can enhance their occupational well-being ${ }^{[14]}$. 


\subsubsection{Marital status}

The study found that the occupational well-being of medical staff in the tuberculosis department who is married is higher than that of medical staff in the tuberculosis department who is unmarried, which may be related to the fact that family stability can provide more social support. This is consistent with the results of foreign studies that married individuals are associated with higher social support.

\section{Conclusion}

The overall level of occupational well-being of the medical staff in the tuberculosis department is not high; in particular, they are associated with the lowest level of physical and mental health. Night shifts, working years, occupational types, and marital status are factors influencing the occupational well-being of medical staff in the tuberculosis department, which reminds nursing managers that they should pay more attention to medical staff who have worked for 6-15 years and provide them with more promotion opportunities. To give more benefits to night shift workers, hospital administrators and the general public should strengthen their respect for the tuberculosis medical staff, especially the tuberculosis nurses, and improve their social status and sense of self-identity.

\section{Funding}

Medical science research key project plan of Hebei health and Family Planning Commission in 2018 (Approval Number: 20180720)

\section{Disclosure statement}

The authors declare that there is no conflict of interest.

\section{References}

[1] Straume LV, Vitters J, 2015, Well-being at Work: Some Differences Between Life Satisfaction and Personal Growth as Predictors of Subjective Health and Sick-Leave. J Happiness Stud, 16(1): 149-168.

[2] Adriaenssensa J, Hamelinkb A, Van Bogaertc P, 2017, Predictors of Occupational Stress and WellBeing in First-Line Nurse Managers: A Cross-Sectional Survey Study. International Journal of Nursing Studies, 73: 85-92.

[3] Sanveingchan S, Phlainoi S, Chai-Aroon T, et al., Nurses Occupational Well-being of Retired Nurses with Productive Aging. Advanced Science Letters, 23: 1019-1023.

[4] Zeng Q, Fu L, Wan B, et al., 2017, A Study on Job Stressors and Mental Health of Nurses in Tuberculosis Department. Chinese Journal of Tuberculosis Prevention, 39(10): 1137-1140.

[5] Hu D, Ma Y, Na L, et al., 2011, Development and Reliability and Validity Analysis of Occupational Well-Being Scale for Medical Workers. Chinese Hospital Statistics, 18(2): 127-129.

[6] Chen L, Liu H, Shang Y, et al., 2016, Preliminary Development and Reliability and Validity Test of Work Well-Being Questionnaire for Hospital Nurses. Chinese Journal of Modern Nursing, 22(15): 2091-2096.

[7] $\mathrm{Hu}$ M, Su CY, Yu ZR, 2017, A Preliminary Evaluation of Occupational Well-Being and Intervention Effect of Nurses in a Public Hospital. Chinese Journal of Modern Nursing, 23(31): 4028-4030.

[8] Tian H, 2010, Survey on Job Satisfaction, Social Support and Subjective Well-Being of Medical Staff in a Special Hospital. Sun Yat Sen University.

[9] Ding C, 2006, Research on the Current Situation and Countermeasures of Doctor-Patient Relationship. 
Shandong University.

[10] Zhuang X, Fu Y, Zhang H, 2017, Qualitative Research on Work Experience of Nurses in Acute Infectious Diseases Department. Evidence Based Nursing, 3(3): 261-265.

[11] Jin X, 2016, Current Situation Analysis and Correlation Research on Nurses' Health Promoting Lifestyle and Occupational Well-Being. Nanchang University.

[12] Zhao Y, Wei L, Sun L, et al., 2017, Analysis on the Status Quo and Influencing Factors of Occupational Well-Being of Nurses in Qingdao Class A Hospitals. Journal of Nursing, 24(21): 4-7.

[13] Li G, Li H, Wang X, et al., 2011, Investigation on Occupational Well-Being of Medical Workers and Analysis of Its Influencing Factors. Medicine and Philosophy, 32(13): 43-44,53.

[14] Gao C, Tong X, 2017, A Survey on the Occupational Well-Being of Clinicians in Urumqi. Journal of Xinjiang Medical University, 40(9): 1209-1212. 